\title{
Jacques Bertin's Legacy in Information Visualization and the Reorderable Matrix
}

\author{
Charles Perin ${ }^{\mathrm{a}}$ and Jean-Daniel Fekete ${ }^{\mathrm{b}}$ and Pierre Dragicevic ${ }^{\mathrm{b}}$ \\ ${ }^{a}$ City, University of London, Northampton Square London, UK EC1V 0HB, London, UK; b Bat. 660, \\ INRIA/LRI, Université Paris-Sud Orsay, 91405, France
}

\author{
ARTICLE HISTORY \\ Compiled June 14, 2018
}

\begin{abstract}
Jacques Bertin's legacy extends beyond the domain of cartography, and in particular to the field of information visualization where he continues to inspire researchers and practitioners. Although in the late $20^{\text {st }}$ century his books were out of print, their re-edition around 2010 has steered a renewed interest and inspired new generations of researchers to re-interpret the principles of Semiology of Graphics and La Graphique in a time of interactive computers. In particular, the work of Jacques Bertin on the reorderable matrix has been very challenging in his time, and the quest to its automation has not been satisfactory to him. This article summarizes Bertin's approach to the reorderable matrix, underlines the limitations of fully automated reordering methods, and introduces Bertifier, a hybrid system to reorder matrices using a combination of machine assistance and human control.
\end{abstract}

\section{KEYWORDS}

Jacques Bertin; reorderable matrices; information visualization; legacy; Human-Computer Interaction; Steerable algorithms.

\section{Introduction}

Jacques Bertin was a French cartographer known for his theoretical work on graphical representations of information. His work had a large influence in areas such as cartography, social sciences and the research field of information visualization, which focuses on what Bertin called monosemic sign systems and is defined as the use of computer-supported, interactive, visual representations of abstract data to amplify cognition (Card et al., 1999).

Jacques Bertin was born in 1918 near Paris ${ }^{1}$. His father was a painter and he was himself skilled at drawing (see Figure 1). He studied geography and cartography at the Sorbonne, then was enrolled in World-War II, for which he later received military distinctions. In 1947 he became research scientist at the Centre National de Recherche Scientifique (CNRS), and seven years later, he founded and directed the Laboratoire de Cartographie at the École Pratique des Hautes Études, now EHESS. In 1974 he became Research Director and his laboratory was renamed Laboratoire de Graphique.

Email: Jean-Daniel.Fekete@inria.fr

${ }^{1}$ This biography and review of Bertin's work is based on a Wikipedia article (Wikipedia, 2017), essays from Palsky (2003), Bord (2011), and Malaurie (2012), and on the authors' visits of the Archives Nationales, the Archives de France, the Bibliothèque Nationale and the EHESS where Bertin's work is preserved. 

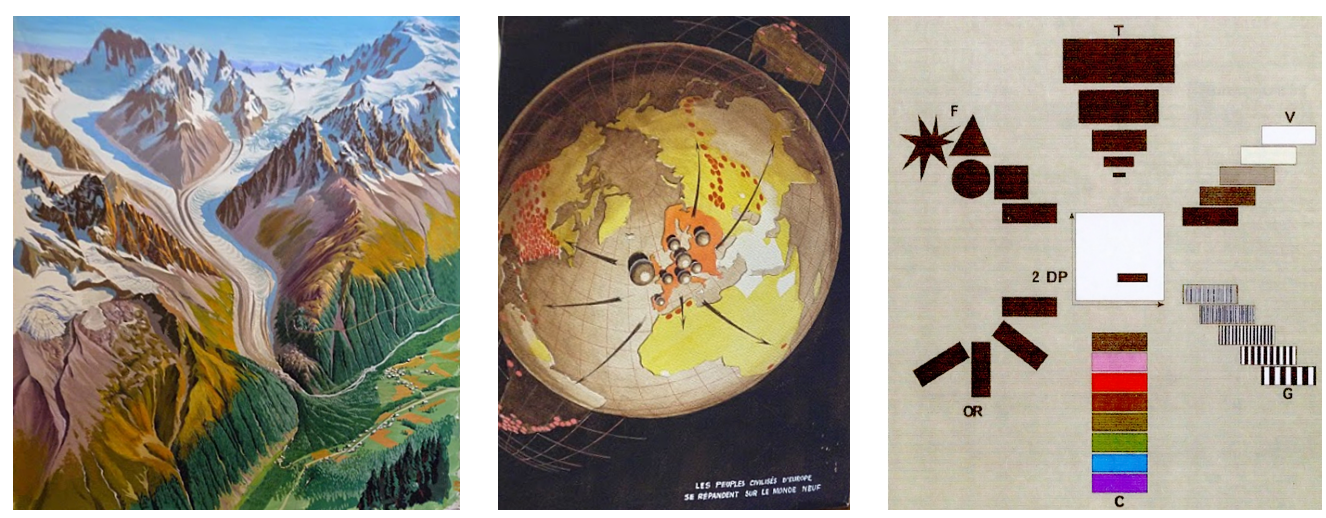

Figure 1. Left and middle: Two schoolbook illustrations hand-painted by Bertin (Bibliothèque nationale de France, Département des Cartes et Plans, Archives Jacques Bertin); Right: Bertin's visual variables (Bertin, 1983) (picture courtesy of Éditions de l'EHESS).

Jacques Bertin retired in 1985. Soon after, his laboratory ceased to exist. During the 1990s he received several important scientific distinctions and continued to publicize and explain his approach to what we now call visualization. He died in Paris on May 3rd, 2010.

\section{The Legacy: Semiology of Graphics}

Jacques Bertin's interests went well beyond cartography, and he soon became obsessed with how to best communicate data visually. This quest was nourished by the need of the social scientists from his institution to understand and convey the data they collected, spatial or otherwise. Bertin's intuition as a cartographer together with years of accumulated experience in advising researchers led in 1967 to the publication of his seminal book Semiology of Graphics (Bertin, 1983). The book is a monumental piece of work with many contributions to information visualization. This work is indubitably the most prominent legacy he left in the field of information visualization. Semiology of Graphics is on the bookshelf of any visualization researcher, is on the reading list of any information visualization course, is praised by both academics and practitioners, has inspired numerous designs and seminal studies, and keeps doing so today.

A central element of Bertin's theory is the idea of visual variable (see Figure 1 right). He identifies eight such variables (two positional variables on the plane and six non-positional visual variables), and describes how to best use them to visually express numerical quantities. Later the framework was studied and refined by prominent researchers in information visualization, including Cleveland and McGill (1987), Mackinlay (1986) and Card and Mackinlay (1997). Bertin also proposed an early taxonomy of network layouts and representations, where we can recognize visual representations that have become popular: arc diagrams, treemaps, 3D treemaps, and adjacency matrices. The book Semiology of Graphics contains a rich variety of original and useful visual representations, many of which have been later rediscovered in the field information visualization, and many others which have not been fully exploited yet. With this work, Bertin was increasingly diving into the representation of abstract data-in contrast to his early work in cartography. 

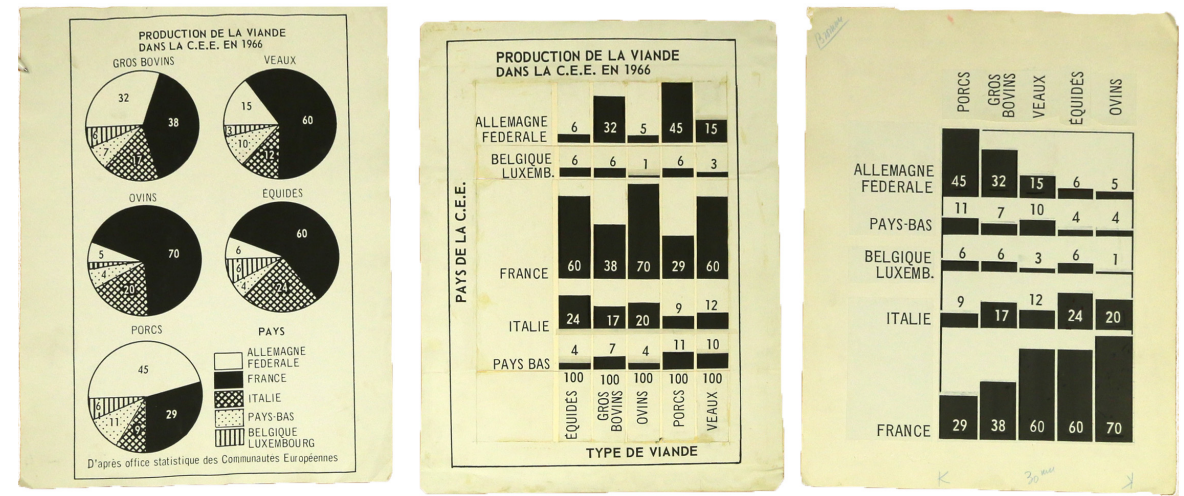

Figure 2. Left: Pie charts showing the contribution of different countries in the production of different types of meat, which Bertin qualified as "useless". Middle: With a matrix visualization, high-level patterns become immediately visible. Right: Since countries and meats do not have a natural order, many other matrices can be produced, including this one, which is more effective. Thus, being able to try different orderings was essential. Drafts for the book La Graphique (Bertin, 1977) Courtesy of EHESS/AN ref. 20010291/36. All rights reserved.

\section{La Graphique}

In 1977, Bertin published another book, La Graphique et le Traitement Graphique de l'Information (Bertin, 1977, 1981). This book is less known to the information visualization community, but to Bertin and his collaborators it elaborates on the most original and important aspects of his work. Some of these aspects were shortly discussed in the 1973 edition of Semiology of Graphics, but they were only fully developed in La Graphique.

The key idea behind La Graphique is that visual representations should be rearrangeable, in order to reveal patterns:

"This is a fundamental point, because it is the internal mobility of the image which characterizes modern graphics. A graphic is no longer 'drawn' once and for all; it is 'constructed' and reconstructed (manipulated) until all the relationships which lie within it have been perceived." (Bertin, 1981)

Bertin explored ingenious mechanisms to facilitate the manipulation of visualizations. One of them, already in use in 1967, is the image file, i.e., papers cards whose top edge served as a visual sorting index, and could be stacked into a compact visualization (Bertin et al., 1967). He also showed how small multiples of maps could be rearranged to facilitate reading, and used families of line charts printed on transparent sheets. Although interaction is now a crucial component in the field of information visualization, Bertin emphasized its importance well before the field was created.

\section{Bertin's Reorderable Matrices}

For Jacques Bertin, many data analysis questions start with a two-dimensional numerical table: one dimension as rows, the other as columns. In Semiology, he lays out a taxonomy of data dimensions, of which one criterion is whether a dimension is reorderable. For example, if a dimension is time, one usually wants to preserve its natural order: it is not reorderable. When both dimensions are reorderable, the appropriate visualization is a matrix, i.e., a table where cell values are encoded visually. For Bertin, the matrix was the most general and clearly the most important visual construction. It was widely used in the Laboratoire de Cartographie.

The archives of the laboratory contains countless paper matrices hand-crafted by Bertin's 

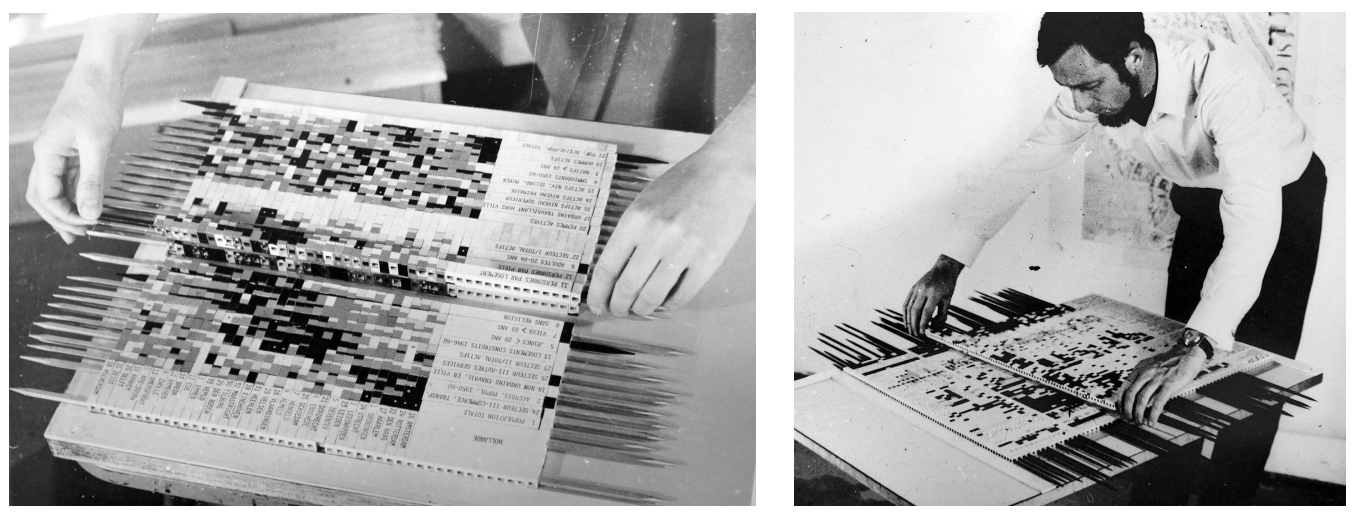

Figure 3. Bertin's collaborator Serge Bonin manipulates matrices of different sizes (Courtesy of EHESS/AN ref. 20150774/21, All rights reserved).

collaborators and students. The most common method involved glue and scissors: matrices were drawn by hand, then photocopied, cut along either rows or columns, reordered, glued, and photocopied again. A simple example is shown in Figure 2. For more complex datasets, the process could take weeks. Once the result was deemed satisfactory, a clean and annotated version of the matrix was produced for publication.

Reordering was clearly the most crucial but also the most time-consuming part of the matrix visualization process. In 1968, Bertin and his colleagues started to design a mechanical device that would facilitate reordering: the reorderable matrix. The device consisted in separate plastic cells, and a rod mechanism for reordering them across either rows or columns (see Figure 3). The device underwent successive refinements up to the 80s, was sold to other French labs, and even used in schools.

Bertin designed and built several versions of the reorderable matrix, each with a different size and visual encoding. In La Graphique (Bertin, 1977, 1981), he explains the general workflow involved in using them. First, an initial numerical paper table was made. Then, cell values were converted into discrete steps on another table. The physical matrix was then assembled by choosing among a collection of physical cells that encode different ranges of values. Then, the matrix was reordered. Finally, meaningful groups were identified and named. The result was then photographed or photocopied, and the final image was used as a figure in the scientific publication or textbook.

\section{From Manual Reordering to Automated to Hybrid}

Bertin and his collaborators employed a diversity of workflows in their analyses. Even in the 1980s, paper and scissors were still used. Nevertheless, Bertin was enthusiastic about the possibilities offered by computers. In 1964, he was already studying computer solutions for rendering and printing thematic maps (Bertin, 1966).

Bertin explored computer solutions to help analysts reorder matrices, but he was limited by the technology available in France at the time. In 1982, he wrote a workshop paper where he describes the functionality a computer program should implement to fully support La Graphique (Bertin, 1982). He also supervised several small-scale software development projects in the 80-90s in order to port his reorderable matrix to computers.

Despite his interest in computerization, Bertin argued against fully automated matrix reordering methods that were developed in other labs, and advocated the key role of manual methods and human judgment in the data analysis process. He was unsatisfied by the output 

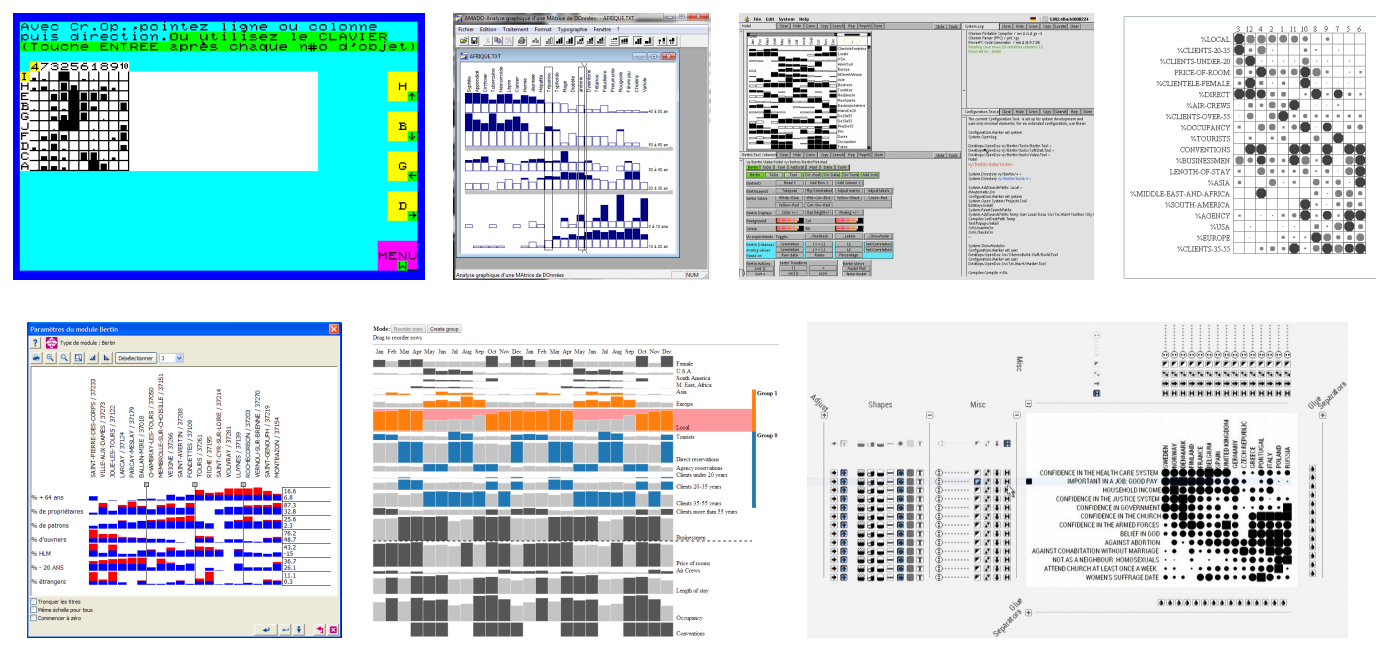

Figure 4. Computer implementations of Bertin's matrices. Top row: MATRIX (Durand and Gimeno, 1987), AMADO (1994), Voyager (Sawitzki, 1996), and Siirtola's reorderable matrix (Siirtola and Mäkinen, 2005). Bottom row: Cartes et Données (2013), Rubin's d3 implementation (2013), and Bertifier (Perin et al., 2014).

of automatic reordering algorithms and argued that automatic methods should be used only to assist humans (Bertin, 1977). To him, "the best graphic operations are those carried out by the decision-maker himself" (Bertin, 1981).

Several other computer implementations of Bertin's reorderable matrix were developed in the last decades, including by information visualization researchers (see Figure 4). However, systems were either rich in terms of functionality but poor in terms of interactivity, or supported smooth interactions but at the cost of only providing a few features. Even systems rich in functionality did not support all aspects of Bertin's workflow. Furthermore, almost all these systems are obsolete now. For an extensive comparison and discussion, see Perin et al. (2014).

To address this gap, in 2014 the authors of this article developed a comprehensive implementation of Bertin's reorderable matrix named Bertifier, based on an thorough examination of his past work (Perin et al., 2014). Bertifier implements features such as computer-assisted interactive reordering, styling and annotation. It relies on recent methods to automatically order tables (Behrisch et al., 2016) that are flexible enough to support human intervention. It is designed to be accessible to a wide audience and is web-based, so it can be used immediately without any installation, while keeping data on the client to ensure privacy. Bertifier is freely accessible at www.bertifier.com.

\section{Conclusion}

Jacques Bertin has been a precursor of information visualization, but his work has not yet been completely revisited and re-interpreted. While Bertin's work has inspired the field of information visualization since its inception, we have realized that his exploratory process for making sense of tabular data using matrices was barely known by the community. By focusing on this specific process, we have been able to directly implement some of Bertin's early thoughts on interaction and data manipulation into a modern interactive tool. Bertifier remains faithful to Bertin's method while leveraging the power of today's interactive computers. It fills a gap between flexible but often tedious manipulations, and fast but often unsatisfactory automatic reordering methods. We hope that Bertin's method of La Graphique will finally become accessible to a wide audience and empower people with data analysis and communication tools 
that were so far only accessible to a small number of specialists. The legacy of Jacques Bertin is still to be explored and will certainly continue to inspire the information visualization community as much as it has inspired the cartographic community.

\section{References}

Behrisch, M., Bach, B., Henry Riche, N., Schreck, T., and Fekete, J.-D. (2016). Matrix Reordering Methods for Table and Network Visualization. Computer Graphics Forum, 35:24.

Bertin, J. (1966). La cartographie statistique automatique. Mathématiques et Sciences Humaines, 17:71-76.

Bertin, J. (1977). La graphique et le traitement graphique de l'information. Nouvelle bibliothèque scientifique. Flammarion.

Bertin, J. (1981). Graphics and Graphic Information Processing. De Gruyter, Berlin. Translation: William J. Berg, Paul Scott.

Bertin, J. (1982). Cahier des charges pour un mini-ordinateur graphique. Actes du second Colloque de micro-info-graphique de Rouen. http://www.aviz.fr/wiki/uploads/Bertifier/bertin-1982-cahier.pdf.

Bertin, J. (1983). Semiology of Graphics. University of Wisconsin Press.

Bertin, J., Lamontagne, R., and Vergneault, F. (1967). Traitement graphique d'une information : les marines royales de france et de grande-bretagne (1697-1747). Annales, 22(5):991-1004.

Bord, J. P. (2011). Jacques Bertin et les Géographes. Point de vue d'un géographe-cartographe. In Ruas, A., editor, Proceedings of the 25th International Cartographic Conference.

Card, S. K. and Mackinlay, J. D. (1997). The Structure of the Information Visualization Design Space. In Proceedings of the 1997 IEEE Symposium on Information Visualization (InfoVis '97), INFOVIS '97, pages 92-, Washington, DC, USA. IEEE Computer Society.

Card, S. K., Mackinlay, J. D., and Shneiderman, B., editors (1999). Readings in Information Visualization: Using Vision to Think. Morgan Kaufmann Publishers Inc., San Francisco, CA, USA.

Cleveland, W. S. and McGill, R. (1987). Graphical Perception: The Visual Decoding of Quantitative Information on Graphical Displays of Data. Journal of the Royal Statistical Society. Series A (General), 150(3):192-229.

Durand, M.-F. and Gimeno, R. (1987). De TMC à CARTAX ou d'un logiciel de recherche à un logiciel d'enseignement. revue Mappemonde, 87(1).

Mackinlay, J. D. (1986). Automating the Design of Graphical Presentations of Relational Information. ACM Trans. Graph., 5(2):110-141.

Malaurie, J. (2012). Hommage à Jacques Bertin, père de la sémiologie graphique. CFC, 212:35-40.

Palsky, G. (2003). L'esprit des cartes: approches historiques, sémiologiques et sociologiques en cartographie. habilitation, Université de Paris XII-Val-de-Marne.

Perin, C., Dragicevic, P., and Fekete, J.-D. (2014). Revisiting bertin matrices: New interactions for crafting tabular visualizations. IEEE Transactions on Visualization and Computer Graphics, 20(12):2082-2091.

Sawitzki, G. (1996). Extensible statistical software: On a voyage to Oberon. Journal of Computational and Graphical Statistics, 5(3):263-283.

Siirtola, H. and Mäkinen, E. (2005). Constructing and reconstructing the reorderable matrix. Information Visualization, 4(1):32-48.

Wikipedia (2017). Jacques Bertin. https://en.wikipedia.org/wiki/Jacques_Bertin. Last accessed Dec 2017. 